UCRL-ID-129679

\title{
Heavy Vehicle Industry Site Visits: Comments from Companies and Conclusions from Technical Committee
}

Rose McCallen, Lawrence Livermore National Laboratory Don McBride and Walter Rutledge, Sandia National Laboratories, Albuquerque Fred Browand, University of Southern California Anthony Leonard, California Institute of Technology Jim Ross, NASA Ames Research Center, Moffett Field

February 1998

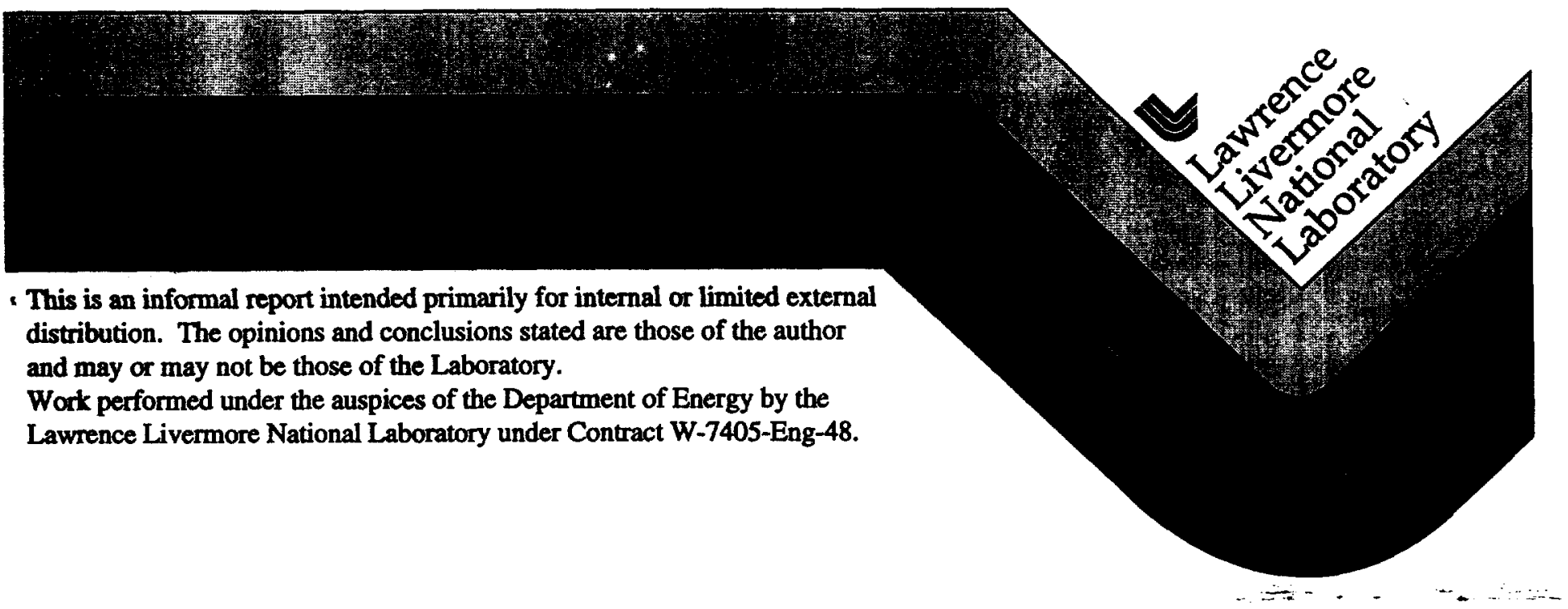




\section{DISCLAIMER}

This document was prepared as an account of work sponsored by an agency of the United States Government. Neither the United States Government nor the University of California nor any of their employees, makes any warranty, express or implied, or assumes any legal liability or responsibility for the accuracy, completeness, or usefulness of any information, apparatus, product, or process disclosed, or represents that its use would not infringe privately owned rights. Reference herein to any specific commercial product, process, or service by trade name, trademark, manufacturer, or otherwise, does not necessarily constitute or imply its endorsement, recommendation, or favoring by the United States Government or the University of California. The views and opinions of authors expressed herein do not necessarily state or reflect those of the United States Government or the University of California, and shall not be used for advertising or product endorsement purposes.

This report has been reproduced directly from the best available copy.

Available to DOE and DOE contractors from the Office of Scientific and Technical Information

P.O. Box 62, Oak Ridge, TN 37831

Prices available from (615) 576-8401, FTS 626-8401

Available to the public from the

National Technical Information Service

U.S. Department of Commerce

5285 Port Royal Rd.,

Springfield, VA 22161 


\title{
Heavy Vehicle Industry Site Visits: \\ Comments from Companies and \\ Conclusions from Technical Committee
}

\author{
Rose McCallen, Lawrence Livermore National Laboratory, Livermore, CA \\ Don McBride and Walter Rutledge, Sandia National Laboratories, Albuquerque, NM \\ Fred Browand, University of Southern California, Los Angeles, CA \\ Anthony Leonard, California Institute of Technology, Anaheim, CA \\ Jim Ross, NASA Ames Research Center, Moffett Field, CA
}

February 1998

\section{Introduction}

This report documents the results of several visits with industry as part of the Department of Energy (DOE), Office of Transportation Technology, Office of Heavy Vehicle Technology, supported Heavy Vehicle Aerodynamics Project. This project is being directed by the DOE representative Dr. Sidney Diamond.

The Project's Technical Team visited the following industrial sites over a three day period from December 9th through the 11th, 1997:

Tractor Manufacturers:

Navistar International Transportation Corporation, Fort Wayne, IN (contact: Gene Olson and Greg Steen)

Freightliner Corporation, Portland, OR (contact: Bill Gouse)

Trailer Manufacturer:

Wabash National, Lafayette, IN (contact: Frank Smidler)

The technical committee members attending all three site visits were Rose McCallen of Lawrence Livermore National Laboratory, Don McBride of Sandia National Laboratories, Fred Browand of the University of Southern California, and Anthony Leonard of California Institute of Technology. We were joined by Jim Ross of NASA Ames for the visit to Freightliner Corporation. Frank Tokarz from LLNL also attended the Freightliner visit.

The purpose of the visits was to

Continue information exchange with industry that began with our January 1997 DOE 
workshop in Phoenix, Arizona, and

Obtain input and comments on the draft Multi-Year Program Plan for Heavy Vehicle Aerodynamics.

The report is divided into two parts; Part I presents the companies' comments and Part II presents the conclusions from the Technical Committee. 


\section{Part I: Comments from Companies}

\section{Overall Summary}

The following is a summary of general comments or recommendations. Comments or recommendations particular to any one site are outlined in the next section.

\section{Design of Aerodynamic Vehicles and Interactions between Tractor/Trailer Manufactures}

Industry wide, there is little interaction between tractor and trailer manufacturers with respect to aerodynamic design. This is due to a large number of factors, primary of which is the very diverse nature of trailer designs. There are a few design constraints on the part of most enclosed cargo trailers (i.e., max height, width, and length, combined with the economic constraints of maximizing interior volume with respect to initial cost while allowing efficient loading and unloading at standard height docks) that enable tractor manufacturers to have a fairly well defined idea of the shape of many of the trailers they can expect to be hauling. However, tractors are trailer type limited depending upon the height of the fifth wheel and king-pin dimensions. Some trailers are set up to be coupled closely to the tractor so the fifth wheel on the tractor must be farther from the cab. Height is also important so that the trailer is pulled level. You might occasionally see a tractor pulling a trailer that is tilted because of a mismatch. Standardization of aerodynamic interface thus becomes extremely difficult. While it appears that this tractor-trailer interface is one area that could benefit from aerodynamic analysis and design, very close interaction with industry would be necessary.

The tractor manufacturers believe they know what is needed for design changes and would rather do the design work themselves, however, they would welcome our thoughts/ experiments on trailer aerodynamics. The only collaborative design, technology development, or problem solving effort that they might consider is one that would give their specific company access to such technology for say 1 to 5 years, before release to their competitors. This type of project would have to be part of a cooperative research and development agreement (CRADA) which is not the objective of this project. All results developed as part of the current project will be made available to all participants.

The trailer manufacturer, on the other hand, admitted that they do not have the resources to do any proactive drag reduction research or development. Any drag reduction changes or additions which they may make are always at the instigation of the customer. They would welcome any help which the DOE may be willing to provide in the area of drag reduction, but only if adequate support (in terms of computational, experimental or field demonstration evidence) were included to enable them to convince their customers of the financial gain to be realized.

\section{Computational Needs}

The tractor and trailer manufacturers pointed out many flow areas of concern, where the effects of design changes on drag are not well understood. It would be beneficial to have a computational tool that can provide such design guidance. For example, the effect on drag due to tractor-trailer and trailer-trailer gap distances, underbody and underhood flows, as well as 
the effects of flow obstructions like handles, mirrors, and sun shades are all of interest. Splash and road spray are safety issues for both truck and trailer manufacturers. Water flow on windows is important, as well as cabin noise due to flow acoustics (exterior noise is secondary).

It was also proposed that the MYPP include the definition of problems involving the flow around generic shapes that exhibit the flow characteristics of heavy vehicles. These problems would be defined by the Technical Team with the assistance of the tractor manufacturers. The generic problems would be modeled with the advanced computational tools and validated with experimental results. An extensive experimental data base for comparison with time dependent LES and vortex method flow solutions would be the result of the project effort. The cases should be complex enough to capture important bluff-body fluid physics, yet simple enough to allow computations to proceed at some realistically high Reynolds number (i.e., high flow velocities). The data base could also be used to evaluate commercial software.

\section{Commercial Software, Computer Resources, and Computational Expertise}

In general, industry has not been successful in implementing Computational Fluid Dynamics (CFD) tools for external aerodynamics. Some success has been achieved for underhood flows. Both of the tractor manufacturers have used computational tools in their tractor designs. However, the general feeling is that existing, commercially available computational tools lack the accuracy required for replacing testing or for demonstrating, to potential customers, any aerodynamic advantages associated with specific design features or proposals. Furthermore, setup, execution, and analysis of results are extremely complex and often beyond the capability of tractor and certainly of trailer manufacturers. Something more advanced and innovative is needed.

It should also be noted that while computing resources may be available on a limited basis, the general computing power available to the tractor manufacturers on a daily basis is limited to the level of a stand alone workstation. It should also be reiterated that while some tractor manufacturers have aerodynamicists on their staffs who are capable of using these tools, the trailer manufacturers do not.

The tractor companies need assistance evaluating the methods used in existing commercial tools. It was suggested that the MYPP include a review of the modeling and numerical approaches used in the commercial software that is currently being considered by the tractor companies. It was also suggested that we work with the commercial software companies, providing them with guidance on how they can enhance their codes to meet the heavy vehicle industry's needs.

\section{Experimental Methods}

The tractor companies are interested in advanced experimental methods and would be interested in the development of the digital particle image velocimetry (DPIV) method for use in their large scale experiments. It was agreed that these methods should be used in the 
experimental analyses of the generic problems.

\section{Demonstration of Trailer Add-Ons}

We were also left with the general impression (from both the tractor and trailer manufacturers) that drag reduction through the use of a boattail does not need to be demonstrated as it is well known that it works. The problem with a boattail lies in unintended consequences caused by its implementation, such as failing to accommodate differing state regulations, maintenance, convenience, and interference with loading, unloading, city driving, or visibility. Thus, if any demonstration is attempted, it should incorporate features to mitigate these other problems and the demonstration should be directed towards proving its usability as well as its effectiveness.

\section{Information Particular to Each Company Visited}

The following are particular comments and suggestions that were obtained from the individual sites visited.

\section{Navistar International Transportation Corporation}

Navistar currently employs $600(+)$ engineers, technicians, and supporting staff. They are in the process of adding 200 to 400 more. They have an aerodynamics group of 2-3 persons.

It was not clear how much design guidance is obtained from computational fluid dynamics (CFD), but a significant effort goes into wind tunnel testing. Navistar has averaged 8 weeks of testing per year; each test supported by 4 people including 2 engineers and 2 modelers. That is about 32 man weeks/year testing--mostly in the wind tunnel at Texas A\&M. The scale is $1 / 8$ or larger.

They have done wind tunnel tests on an unmodified and modified, truck mounted system which was mounted on the floor of the tunnel and they have given the geometry and test conditions to two software companies to see if they could produce correct results without knowing the answer. One company which uses a traditional CFD approach (finite volume, structured/unstructured grid, Reynolds-Averaged Navier-Stokes (RANS)) has yet to be heard from. The other company using a different approach (a lattice gas method) shows promise, but was still unable to duplicate the wind tunnel results. Navistar thinks that part of the problem is that the boundary layers are laminar or transitional in the tests but that the codes may assume turbulent boundary layers. At the present time, they are not happy with CFD results.

They are intrigued with the lattice gas method because (1) the setup time is much shorter (irregular grid cells at the body surface in a set of otherwise cubical grid volumes are used), (2) the method seemed to do a good job on an Ahmed slant add-on's for base drag reduction, (3) some automotive companies are showing high degree of interest in this CFD technology and, (4) the method is refreshingly new to Navistar.

From the proposed DOE project they would ultimately like to get access to robust, accurate CFD tools with reasonable setup and run times. If they don't acquire their own CFD group they might be interested in leasing time at an outside computational facility, much like how they lease time at a wind tunnel. The CFD facility would need to have the necessary software and access to appro- 
priate computer hardware. The facility would also need the expertise available to setup the computational runs and to postprocess the data. (The analogy with a wind tunnel operation seems to be a very good one.)

Navistar was extremely strong in their criticism of Project 1 . They felt that existing computational tools are inadequate for their purposes, although they did hold out hope for the lattice gas computational method which they feel has progressed significantly within the past year. There was no interest in conducting additional trials of a boattail geometry. They don't want us (National Labs and universities) getting involved in design. They already know how to reduce the drag on trailers. Boattail plates will provide 7 to $14 \%$ and adding skirts will provide another $10 \%$ for a total reduction approaching $25 \%$. Thus they don't believe the demo project with a boattail (Project 1, Part 2 of draft MYPP) is useful. At the present time, the customers don't want to deal with these add-ons.

As for the gap between tractor and trailer -- if the gap is filled half way, one achieves $80 \%$ of the drag reduction of filling the gap completely. This is something that is already done. Navistar, as well as their competitors, install cab/sleeper rear extender/fairing as part of the Aero packages. These devices effectively close the gap from 35 inches to an effective gap of 18 inches. The result is that the amount of drag associated with gap flow is reduced $80 \%$. What is really needed is the ability to close the gap completely; top, sides, and bottom. This is where the challenge lies.

Small changes in the tractor configuration can significantly alter the overall drag coefficient at yaw. For example, significant drag increases ranging from 2 to $10 \%$ can result from items such as protruding grab handles, protruding turn signals, and mirrors.

Navistar believes that it is important for the technical team to become very familiar with truck aerodynamics issues, like the ones mentioned above, before the team can develop appropriate CFD software. It was suggested that the team spend some time developing an understanding of truck flow characteristics. Frequent visits to Navistar are welcomed, and Navistar is open to the idea of working together to define generic flow problems of interests that the team can fully investigate computationally and experimentally.

Fred Browand showed some DPIV measurements taken of the flow around an axisymmetric Stratford body. They seemed impressed that such a capability might be routinely available in a few years. The initial cost for setting up such a system now would be about $\$ 120 \mathrm{~K}$. In a few years, systems like this might be routinely available in wind tunnels to provide much more comprehensive information.

Navistar mentioned the need for improved representation of the ground plane in experiments. A concept developed by John Foss of Michigan State University may be of interest here. The magnitude of the Foss' proposed facility would probably require a more significant investment than is being proposed for this project. Navistar recommends that the MYPP have provision for inputs from people such as John Foss, Georgia Tech Research Institute (blowing and suction devices), and others.

\section{Freightliner Corporation}

Freightliner has an analysis group with 1-2 aerodynamicist and an experimental group with 1-2 
aerodynamicist. The analysis group's primary focus is structural. In addition, Freightliner has close ties with Daimler Benz. Freightliner appears to make limited use of CFD. The CFD results shown were generated by commercial software companies.

Aerodynamic changes are mostly by trial and error with wind-tunnel and coast-down testing. Freightliner does the majority of its wind tunnel testing in Germany. Their suppliers often use different facilities. They would like the opportunity to use other facilities close to their headquarters. The level of effort for aerodynamic testing and development has large variations based upon the product and problem being studied.

Emphasis on underhood flow has produced improvements in fuel and engine cooling efficiency. We were shown some CFD results used to guide underhood design. These results were coupled fluid/heat transfer simulations.

Freightliner would be interested in a CRADA type of agreement where design work would be held as industry proprietary for 1 to 5 years. They would also support a non-proprietary project (if the subject was appropriate) that was set up as a consortium through SAE or the Truck Manufacturers Association. One topic might be spray reduction. Something like this might need to be a joint DOE and DOT/NHTSA project as both fuel economy and safety would be addressed.

\section{Wabash National}

Wabash employs about 4500 people in Lafayette, Iowa, and Tennessee, and builds about 50,000 trailers per year. It has about $20-25 \%$ of the total business. Wabash has a staff of about 40 engineers -- mostly doing design and static structural testing.

Trucking (fleet) companies (and therefore trailer manufacturers) have to deal with myriad regulations that might vary from state to state. The trailer maximum length is 53' for most states and all interstate highways. Some states allow two 45' trailers and some Western states allow three 28' trailers. The maximum length from kingpin to rear axle varies so that trailers are outfitted with a sliding suspension for the rear axle. The total weight limit is $80,000 \mathrm{lbs}$, but the load distribution may vary from state to state. A typical trailer weighs about 14,000 pounds and a tractor about 18,000 pounds, leaving about 48,000 pounds for cargo. The weight distributions is 12,000 pounds on the front truck axle, and 34,000 pounds total on the two rear axles.

There is a 5" radius on the sides at the front of the trailer -- all other corners are sharp.

The traditional aluminum siding includes exterior aluminum vertical posts that are 8 " wide and $3 /$ 8 " thick. with perhaps 20 total at various spacings. These clearly provide considerable roughness. In a wind tunnel test, an extra post was added by mistake close to the leading edge. The next post was 12 " downstream. The extra post yielded a $4 \%$ drag penalty.

More recently, a composite siding is being used. This new siding consists of thermoplastic sandwiched between two steel sheets (called DuraPlate by Wabash). This is a smoother siding but still contains many $1 / 4$ " rivets that protrude about $1 / 8$ " onto the outside. The rivet heads (rounded ends) are placed on the inside to make the inside smoother for cargo handling. 
The space between the floor and the road is very messy aerodynamically. Underneath the floor are 4 " I-beams spanning the width of the trailer and spaced 12" apart. In front is the landing gear with supporting structure. At present, no attempt is made to shape the supports for aerodynamic purposes. The payoff for the extra cost is unknown. The incoming flow is probably quite dirty anyway because of the proximity to the rear wheels of the tractor. The aerodynamics of the rearwheel system is made worse by the presence of the sliding suspension mentioned above as well as brake chambers, air tanks, valves, etc. Adding skirts has been tried by trucking companies but damage during use was too costly.

Wabash builds a RoadRailer trailer which contains a tongue and slot at opposite ends. The trailers can be attached front-to-back with a rail bogie at each end, and put directly on the railroad track. In this configuration, the gap between trailers is about 1 ' and the slack per truck is a fraction of an inch.

Wabash does no aerodynamic design or testing, and neither do any of the other trailer manufacturers. They would be happy to have help on aero issues. Wabash would like to have tools available to estimate changes in drag coefficient or fuel savings that would result from design changes so that a reliable estimate of the net benefit can be made. However, they indicated they are not willing to invest in the expertise required to run such codes, so it can be assumed that any benefit from advanced tools would be on a 'buy from commercial software companies' basis and then only if the customer were willing to pay for the analysis. Thus, he supports the efforts in the draft MYPP.

The aero boattail (flat plates extending from rear of trailer) tested by Ross \& Olson was tested over-the-road by a fleet customer of Wabash. Tests demonstrated fuel savings (to Schneider's satisfaction, but how much I don't know), but the configuration was deemed impractical from an operations/maintenance point of view.

They indicated that keeping drivers is a major problem with the big haulers, so driver satisfaction with the job is a major consideration and could be a factor in determining acceptability of add-on devices that caused major inconvenience to the driver.

\section{Facts about Heavy Trucks}

There are Federal and State regulations concerning lengths, weights, width, etc., because so many states are grandfathered to existing laws. Included in this section is a 'rough' list of limitations to give the reader an idea of the length and weight limitations. For example, there are no overall length regulations on interstate highways but there are regulations on State highways. The Federal law states that on interstate highways the semi-trailer must be at least 48 feet, no less. Most trailers are 53 feet, but it's not a limit. For the actual Federal and State regulations one should consult the U.S. Size and Weight Regulation (CFR 23 Part 658).

The following is a 'rough' list of facts on heavy trucks:

Weight

Single tractor-trailers are limited to $80,000 \mathrm{lbs}$ gross weight. This is a Federal limit. 
Axle load limits are $12,000 \mathrm{lbs}$ front axle, $34,000 \mathrm{lbs}$ total on the two rear axles.

Empty weight is about 14,200 lbs for a 53 foot trailer, 18,000 lbs for a tractor - therefore payload is about $48,000-50,000 \mathrm{lbs}$.

Size

$53^{\prime}$ length $\times 13^{\prime} 6^{\prime \prime}$ height $\times 1023 / 8$ " width

Doubles can be $48^{\prime} \times 48^{\prime}$ in length (in a few states only on designated routes)

Triples can be $28^{\prime} \times 28^{\prime} \times 28^{\prime}$ (allowed in some western states only)

Costs

Trailers cost in the range of $\$ 14 \mathrm{~K}$ to $\$ 18 \mathrm{~K}$ (about $\$ 1$ per pound)

Tractors cost in the range of $\$ 80 \mathrm{~K}$ to $\$ 120 \mathrm{~K}$ (about $\$ 5-\$ 6$ per pound)

Fuel cost is approximately $25 \%$ of total operating costs

Numbers

230,000 trailers manufactured in US each year

The life time of a trailer is about 10 years, so there are probably between $2-3$ million trailer in the US.

There are probably 2-3 trailers for each tractor. 


\section{Part II: Conclusions from Technical Committee}

\section{General Comments}

The purpose of the DOE Heavy Vehicle Aerodynamics Project is to use government resources to bring the aerodynamic expertise available in government organizations and academia to bear in assisting the heavy vehicle industry to reduce aerodynamic drag on trucks. The obvious payback from this investment is the reduction in fuel usage and derivative reduction in the U.S.'s dependence on foreign oil imports.

With reference to the draft MYPP, Sid Diamond of the DOE Office of Transportation Technology, Office of Heavy Vehicle Technology was enthusiastic about both Project 1 with near term payoff by the use of existing tools and a demonstrative project, as well as a longer term effort to develop and utilize more advanced computational tools in Project 2. It is the opinion of the Technical Team that, after the industry site visits, this original plan of a two-fold attack on the problem still seems to make the most sense. Project 1 attacks the problem with current, unique capabilities and is directed toward the area most in need of help - trailer aerodynamics, while Project 2, a longer range effort, is directed toward developing new capabilities needed to more accurately model the flow regimes we know to be important in heavy vehicle aerodynamics. However, modifications to Project 1 and expansion of Project 2 should be made to reflect the recommendations and guidance provided by industry.

Project 1 was oriented toward helping the trailer industry. We heard from both tractor manufacturers we visited that direct tractor design assistance is not needed at this time and that they are not directly involved in trailer design. The trailer manufacturers do not have any aerodynamics capability and would welcome aerodynamic design help. One negative comment on the MYPP from one of the tractor manufacturers had to do with the demonstration of the particular ogive boattail designed and tested by Sandia. We have been asked by DOE 'what exactly stops truckers from using trailer add-ons'. While not talking to a fleet representative, we did hear that a type of boattail consisting of relatively shallow panels called vortex plates has been used by one fleet operation and that it did reduce drag - how much, we didn't hear; but its use was discontinued due to unintended consequences. This says to us that the total problem may not have been well defined and, therefore, the vortex-plate configuration, as installed, was an inadequate solution because it only dealt with a part of the problem. We believe that it may be possible that with a much closer interaction with both the trailer manufacturers and the fleet operators, this and other trailer aerodynamic problem areas could be addressed and satisfactory solutions found and demonstrated in Project 1 .

The goal of Project 2, to develop new tools and/or modeling methods, is also an important goal in this project. While it is necessary to have industry 'buy-in', we don't feel that industry has the expertise to correctly identify the proper path in this development. The technical committee has significant expertise and years of experience in fluid dynamics modeling and, through continued consultation with other experts at the National Labs and academia, should be able to evaluate possible 'value returned' from different development paths (i.e., gridless methods vs. structured grids vs. unstructured grids or finite element vs. finite difference vs. vortex approaches or even direct numerical simulation (DNS) vs. large-eddy simulation (LES) vs. Reynolds-averaged Navier- 
Stokes (RANS) modeling of turbulent flows). When we speak of 'value returned', we refer not only to fidelity of simulation or accuracy of results but also to a reasonable expectation of achieving usefulness on a design scale with 'workstation' hardware by less-than-research-class aerodynamicists. Thus, it seems unwise to us to 'totally scrap' the RANS approach in favor of a total commitment to LES when virtually all of the present production class computational fluid dynamics (CFD) codes use the RANS approach (i.e., the vast majority of the aerospace and automotive communities) and LES still has some development required to be capable of being used on complex fully-integrated applications. However, a significant amount of recent development indicates that the LES method might very well provide higher fidelity simulation of the large scale, timedependent flow structures found in truck aerodynamics. Thus, we believe that the two-pronged parallel approach (RANS and LES) involves much less risk while still retaining the potential capability of long-term payback from the LES approach. As the work proceeds, annual reviews will determine the continued viability of expending resources on any of the computational approaches (LES vortex methods, LES finite element methods, or RANS finite difference/volume methods). This viability will be defined in the context of the 'value returned' described above.

\section{Overall Conclusions}

The following are general conclusions of the Technical Committee as to how the MYPP should be modified based upon the site visit notes and the above comments.

\section{Redefine Project 1 in the MYPP.}

The stated purpose of Project 1 was to provide near-term impact through emphasis on existing tools and capabilities and to focus on the trailer drag problem. None of our discussions or conclusions prove this is the wrong way to go - only that the job may be more difficult than originally anticipated.

Project 1 involved the use of existing computational and experimental tools to improve trailer aerodynamics and to demonstrate a previously tested boattail design.

a. In view of the tractor manufacturers' observations that trailer flow (and therefore drag) is influenced strongly by the shape of the tractor coupled with the fact that there is currently very little interaction between the tractor and trailer manufacturers in the area of aerodynamic design or analysis (see item 1 of the "Overall Summary" section of the site visit notes), it is suggested that a strong component of Project 1 should be to: 1.) verify this tractor/trailer interaction experimentally and, 2.) quantify the extent to which existing computational tools are able to predict (or fail to predict) this interaction, and why.

b. Due to the lack of aerodynamicists in the trailer manufacturer community, their desire for assistance, the large percentage of the drag that comes from the trailer, the aerodynamics expertise and tools (both computational and experimental) that exist within the DOE, NASA, and the academic community, coupled with the DOE's desire to fund efforts that will result in trailer drag reduction, it would seem logical that another strong component of Project 1 should involve an effort to help the trailer community attempt to improve trailer aerodynamics, providing substantiation of aerodynamic results so they can "sell" those solutions to their customers (i.e., fleet operators).

c. Since the negative impacts, or usage consequences, of a large solid or inflatable boattail, such as that tested by Sandia, are generally felt by industry to outweigh the resulting fuel 
savings (see item 5 of the "Overall Summary" section of the site visit notes), any demonstration effort should address ALL of the impacts (i.e., not just drag reduction) resulting from any trailer modifications. Our goal in any demonstration project would be to demonstrate that computational fluid dynamics tools can provide useful design guidance when used properly. A model problem will be used to demonstrate this in Project 1. One current suggestion is to consider base mounted vortex plates instead of the Sandia designed boattail as originally proposed and to install them in such a way as to minimize unintended consequences.

d. Since tractor manufacturers have found existing commercial codes to be largely inadequate to simulate the flow fields of interest, investigation of the regimes of validity of these commercial codes along with a knowledgeable assessment of the validity of other existing, but still developmental, codes would be very valuable in the near term.

\section{Expand on Project 2}

The stated purpose of Project 2 was to provide the tools necessary to accomplish the longer term goal of a fully-integrated, aerodynamic tractor-trailer combination.

Discussions left us with no major reason for substantial modification to the direction Project 2 should take. It should still attempt to deliver usable and accurate software to the trucking industry within a reasonable time. This approach should still be to improve computational methods that could be used for the design of a fully-integrated, heavy vehicle highway transportation system.

However, there does seem to be a necessity to expand the scope of Project 2 in order to support these goals. First, we should define a set of "generic problems", the complexity of which would increase as the computational codes become more capable. The cases should be complex enough to capture important bluff-body fluid dynamics, yet simple enough to allow computations to proceed at some realistically high Reynolds number (i.e., high enough to trigger the flow elements being modeled, yet low enough to allow computations to be made within reasonable machine times). These generic problems would be modeled with the advanced computational tools and validated with experimental results. The quality and completeness of this experimental database becomes of utmost importance for the following reasons. Detailed, high fidelity code validation efforts will ultimately require detailed, time-dependent velocity field data as well as accurate pressure boundary data to support their development. No matter which computational model is used, data at full-scale Reynolds numbers is required to establish the proper transition criteria and turbulence models to be used in the various flow field regimes found on full-scale trucks. The database could also be used by the commercial software companies to evaluate the use of their software. 